\title{
Ira, fibromialgia y ansiedad: aproximación terapéutica desde un CSM
}

Cada época tiene sus neurosis y cada tiempo necesita su psicoterapia.

Victor FRANKL (2)

RESUMEN: Mostramos un programa de tratamiento grupal de la fibromialgia realizado en un centro de salud mental.

PALABRAS CLAVE: Fibromialgia, sobrecarga emocional, ansiedad, ira.
SUMMARY: We show a group treatment program of fibromyalgia done in a mental health facility.

KEY WORDS: Fibromyalgia, emotional surcharge, nervous tension, rage.

Independientemente de que partamos de un enfoque etiológico de la fibromialgia de tipo biológico, o lo entendamos como un trastorno somatoforme o incluso perteneciente al espectro conversivo, nos encontramos con la realidad clínica de que estas pacientes son multiconsultantes de los servicios de Atención Primaria, Reumatología y Salud Mental, por lo que nos vemos obligados a dar una respuesta psicoterapéutica a su demanda.

Es cierto que, en nuestra experiencia, encontramos que en un porcentaje elevado, aproximadamente en un 30\%, el diagnóstico de fibromialgia está enmascarando conflictos sociolaborales («rentismo»), pero este mismo porcentaje se puede encontrar también en otros trastornos de tipo adaptativo o en los síndromes ansioso-depresivos que tanto colapsan los centros de salud mental y atención primaria, por lo que este sesgo rentista no debería ser un criterio de exclusión para tratar clínicamente esta afección. Por otro lado, encontramos que verdaderamente un $70 \%$ de estas pacientes presenta un sufrimiento psicoemocional y físico que debe ser abordado psicoterapéuticamente (3).

Sabemos que abordar este tipo de patologías puede favorecer la sobrediagnosticación e incrementar la demanda, pero «el daño ya está hecho», debido a la gran expansión social de dicho diagnóstico y que ha sido ampliamente aceptada la «existencia» de la fibromialgia como enfermedad; así que nos vemos con la necesidad de «aceptarla» como demanda legítima y de construir técnicas eficaces de intervención para disminuir el malestar que padecen dichas pacientes y sus familiares (4).

En el presente trabajo huimos de polemizar sobre el aspecto nosológico y sociosanitario de esta patología, para ello remitimos a los excelentes artículos ya publicados en esta revista por Javier Ramos García (5) y Guillermo Rendueles Olmedo (6) de cuya visión nos sentimos muy cercanos, básicamente queremos mostrar la descripción de la intervención que hemos desarrollado durante los últimos cinco años en 
respuesta a la necesidad de atender a estas pacientes y sus familias. También queremos mostrar cómo a través de la medición del efecto de nuestra intervención clínica hemos abierto nuevas posibilidades de comprensión e investigación sobre la relación entre la modulación de las emociones, específicamente de la modulación de la ira, con la probabilidad de padecer fibromialgia. Así, aunque nuestra primera intención no era realizar una investigación, podemos mostrar los hallazgos obtenidos a partir del análisis de la intervención clínica que realizamos.

\section{Fundamentación teórica del programa de tratamiento de la fibromialgia}

Aunque somos más partidarios de una concepción sistémica y constructivista de las enfermedades mentales (en tanto que entendemos que cada persona construye su propia realidad en el marco de los diferentes contextos relacionales de los que forma parte), incluso en relación con la fibromialgia se podría hablar de construccionismo social, volviendo a hacer alusión al artículo publicado por Javier Ramos García, asumimos que el modelo Bio-Psico-Social se muestra muy práctico y clarificador a la hora de explicar enfermedades de «nueva aparición», además no consideramos incompatible ver este modelo desde un punto de vista sistémico-constructivista $(7 ; 8 ; 9)$.

Así, asumiendo una etiología multifactorial y partiendo de un modelo BioPsico-Social, en la figura siguiente mostramos los aspectos que consideramos implicados en la construcción de la fibromialgia; los hemos encontrado a partir del discurso de nuestras pacientes. Con este gráfico no se pretende dar una explicación completa y clínica del síndrome sino que es un «mapa», elaborado progresivamente a partir del trabajo psicoterapéutico con las pacientes, y que nos sirve de guía para el desarrollo de la intervención: más que ser una explicación etiológica de la enfermedad es el resultado de la «deconstrucción» de la fibromialgia a través del proceso terapéutico realizado conjuntamente con las pacientes. Con el fin de que resulte más clarificador, intentaremos desarrollar y justificar por qué hemos considerado cada uno de los conceptos presentes en este mapa explicativo:

- Vulnerabilidad somática: Existencia de una vulnerabilidad biológica que determina la respuesta individual ante acontecimientos estresantes y desbordantes. Hace referencia a la teoría de la vulnerabilidad-estrés, cada individuo posee una tendencia a sufrir somáticamente un tipo de enfermedad.

- Burn-out familiar: hemos adaptado el término del burn-out al ámbito de la vida familiar después de escuchar los relatos de una proporción significativa de pacientes que mostraban realmente situaciones de sobrecarga familiar que se merecían dicha etiqueta. Los siguientes ejemplos de las descripciones de nuestras pacientes son una muestra de la dimensión y la legitimidad de incluir este término: «Mi marido me hacía subir cuatro pisos para atarle el cordón de 
los zapatos porque él no podía debido a su sobrepeso»; «los lunes empiezo a trabajar casi más agotada que al final de semana porque los fines de semana tengo a mis cuatro nietos en casa porque mis hijas me lo piden. Ellas necesitan descansar y disfrutar de su tiempo libre»; «después de años de convivir con mis suegros tuve que hacerme cargo del Alzheimer de mi madre». Todos los profesionales de salud mental y atención primaria estamos acostumbrados a este tipo de quejas que denominamos «victimistas» y que en el caso de pacientes fibromiálgicas son muy frecuentes, pero, realmente en estos casos dichos relatos describen cuotas tan altas de sobrecarga que pensamos que es adecuado utilizar el término de «síndrome de estar quemado» ya que se amolda mucho mejor a sus vivencias y narrativas que el de «victimismo».

- Burn-out laboral: encontramos un porcentaje importante de pacientes que junto a la carga familiar habían trabajado en profesiones que requerían esfuerzo físico importante, o bien por el exceso de horas que dedicaban al trabajo o bien por la actividad física que su profesión requería: limpieza, mercado, supermercados, peluquerías, hostelería, etc. Por tanto, en el caso de la fibromialgia y, evidentemente también valdría para la distimia, podemos hablar de burn-out multiplicado por dos.

- AVES: las pacientes relatan sucesos traumáticos que funcionan como desencadenantes de su malestar físico, generalmente duelos: muerte de un hijo, de una madre, separación conyugal o algún tipo de accidente con daños físicos inicialmente leves o moderados. También aparecen situaciones en la infancia de intenso sufrimiento emocional: abandono por parte de los padres, problemas económicos importantes en familias de origen por los que se veían obligadas a asumir responsabilidades de forma temprana (cuidado de hermanos pequeños, cuidado del hogar, etc.) con las consiguientes carencias en cuanto a las necesidades propias de la edad.

- Disarmonía relacional: en un gran número de casos detectamos relaciones disarmónicas con la pareja, hijos o con familias de origen: «A los 18 años me fui de casa, no podía vivir más con mis padres, ellos nunca se mostraban satisfechos. No pude estudiar porque me obligaron a trabajar en el negocio familiar y nunca tuve un sueldo porque según ellos ya me pagaban bastante manteniéndome. Ahora tengo a mi madre en casa, tiene demencia, y necesita tantos cuidados como una niña pequeña». En muchos casos aparecen dificultades en la relación de pareja que muchas veces quedan al descubierto al iniciarse los síntomas que exigen un cambio de posición e involucración de los cónyuges o familiares cercanos.

- Déficits afectivos: evidentemente una consecuencia de la disarmonía y sobrecarga familiar es la vivencia de falta de estímulos afectivos en la vida cotidiana, también algunas pacientes relatan carencias afectivas desde la 
infancia, en el otro extremo también encontramos casos donde aparecen relatos de la infancia con un vínculo excesivamente simbiótico o una sobrecarga afectiva con idealización de la relación parental que ha favorecido que se cree un yo frágil que fácilmente se siente frustrado y desatendido por los demás y desbordado por las situaciones estresantes.

- Rasgos de personalidad: es frecuente que nos encontremos con personas emocionalmente dependientes, que presentan dificultades para comportarse asertivamente y que, generalmente, se identifican y actúan en función de roles rígidos de funcionamiento relacionados con el «ideal» que se presupone a la figura femenina, aspectos que deben ser abordados desde una perspectiva de terapia de género. Se caracterizan por ser excesivamente responsables y autoexigentes, este estilo de funcionamiento superyoico dificulta el cambio ya que fácilmente se sienten culpables o adoptan una posición «victimista», es decir «no asertiva», cuando se proponen delegar en los demás o buscar ayuda para evitar la sobrecarga de obligaciones y responsabilidades. Este aspecto ha sido destacado casualmente en nuestra intervención-estudio al medir la modulación de la ira de estas pacientes a través del Inventario de la Expresión de la Ira Estado-Rasgo (STAXI-2), que a raíz de los datos obtenidos ha proporcionado una creciente relevancia en nuestra aproximación al síndrome y el cual describiremos más detalladamente.

Este mapa de los componentes psicosociales inferido a partir de las narrativas de las pacientes ha guiado el foco de nuestras intervenciones estructurando módulos diferenciados que se han trabajado en cada sesión.

\section{MODELO PSICO-BIO-SOCIAL}

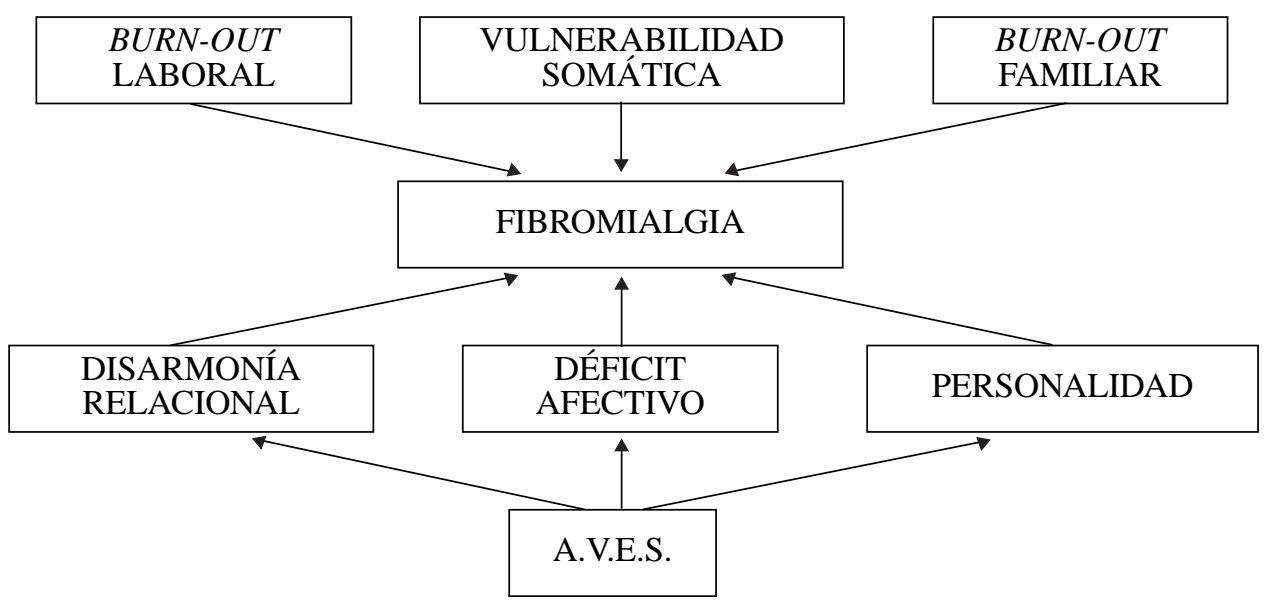


DEBATES E INFORMES

\section{Metodología}

Optamos por la intervención grupal para optimizar recursos y aprovechar los factores terapéuticos e interpersonales que favorece el setting grupal, así como también evitar la dependencia asistencial que se puede dar en estos casos (10). El programa de intervención grupal consta de diez sesiones, de hora y media de duración que se realizan quincenalmente. Se lleva a cabo en co-terapia, siendo el psicólogo del CSM el terapeuta principal y la enfermera del mismo centro la co-terapeuta.

Es un grupo cerrado. Las pacientes que, durante estos años, han participado en cada grupo son de 8 a 15. Tienen un diagnóstico de fibromialgia, en algunos casos también han sido diagnosticadas de trastorno de ansiedad y trastorno depresivo; o bien ya están en tratamiento individual en el CSM o bien son derivadas directamente por Reumatología o Medicina de Familia de nuestro sector específicamente para la intervención grupal.

A lo largo de nuestra experiencia hemos ido ampliando el foco de intervención de nuestro programa. En un inicio nuestro principal objetivo era conseguir reducir la ansiedad, a través de técnicas de relajación y otras técnicas aplicadas en grupo, entendiéndola dentro de un proceso cibernético-circular, es decir, ansiedad como respuesta reactiva al dolor y a la vez como generadora o intensificadora del mismo. Para medir el nivel de ansiedad y el efecto de la intervención pasamos al inicio y al final del programa el Cuestionario de Ansiedad Estado-Rasgo (STAI).

Con el tiempo introdujimos la medición del nivel de expresión de ira con el Inventario de Expresión de Ira Estado-Rasgo (STAXI-2), por ser una emoción que aparecía reiteradamente en las sesiones y que seguía el mismo funcionamiento circular que la ansiedad, reacción al dolor y generadora del mismo, como resultado de la tensión muscular que genera la ira contenida. En este sentido los resultados obtenidos en las escalas del test STAXI-2 nos llevó a formular nuevas hipótesis sobre la importancia de la inadecuada expresión emocional de la ira en la «construcción» del malestar de nuestras pacientes y nos hizo pensar que este test podía ser muy útil para corroborar el componente somatizador o incluso conversivo que se da en dicha afección.

Desde estos aspectos más intrapsíquicos fuimos ampliando el foco a los procesos relacionales que se detectaban en el discurso de las pacientes hasta encontrarnos con la necesidad de realizar una sesión grupal invitando a las parejas o personas más significativas que también «sufrían» el peso de la fibromialgia.

Los objetivos generales del grupo basándonos en el mapa que hemos descrito antes se dividen en los siguientes módulos que según el grupo podían ser trabajados en dos o más sesiones: a) aprendizaje de técnicas de relajación muscular; b) aprendizaje de habilidades de afrontamiento y control de la ansiedad; c) habilidades para aumentar capacidad de adaptación a situaciones de sobrecarga o estrés 
mediante resolución de conflictos; d) habilidades para aumentar la tolerancia a la frustración y asertividad; e) aumentar la calidad de las relaciones personales y la expresión emocional adecuada.

Para la consecución de éstos, el programa consta de aproximadamente diez sesiones. Las técnicas utilizadas a lo largo de las sesiones derivan de la terapia familiar sistémica (re-historiar el ciclo de la pareja, detectar interferencias de la familia de origen, redefinición de los roles jugados en la relación de pareja, ritual de reparación de daños emocionales, tareas y compromisos para el cambio), de la psicoterapia estratégica breve (relajación sugestiva de escenas agradables, sugestiones metafóricas: «limpieza interna», «liberarse de cargas, sacarse un peso de encima», distintos tipos de relajación muscular), y de la psicoterapia breve cognitivo-dinámica (redefinición positiva de acontecimientos vitales estresantes, connotación positiva de roles de género, concienciación de distorsiones cognitivas y creencias desadaptativas, señalización de mecanismos de defensa, prescripción de cambios conductuales y relacionales).

\section{Descripción de las sesiones}

A continuación describiremos muy esquemáticamente cada una de estas sesiones tomando como referencia uno de los grupos que hemos realizado:

- Primera sesión: Se dan las explicaciones y compromisos para crear el setting grupal y se pasa a la presentación individualizada y personalizada de los componentes del grupo. Introducimos los conceptos básicos sobre nuestra visión de la fibromialgia realizando una primera intervención psicoeducativa general y planteamos los objetivos generales del grupo.

Realizamos ejercicios ideo-motores para demostrar las reacciones musculares al estrés y a las cogniciones negativas. El objetivo es aumentar la conciencia de las reacciones tensionales y musculares que todas las personas sufren al pensar en situaciones estresantes o desagradables, explicando que este tipo de tensiones inconscientes también se dan al afrontar e intentar adaptarse a las dificultades diarias.

Explicación del ciclo dolor-ansiedad. La tensión inconsciente y automatizada frente a los estímulos ansiógenos, entre éstos también consideramos el propio dolor, aumenta la tendencia a sufrir dolores.

Explicación de la vulnerabilidad cuerpo-mente: se introduce el concepto de umbral del dolor determinado principalmente por dos componentes que explicarían las diferencias interindividuales en la percepción del dolor: componente biológico y componente emocional, «carga vital». Focalizamos el trabajo grupal hacia este segundo componente. Presentación de los beneficios terapéuticos de la 
DEBATES E INFORMES

relajación, tanto en los planos muscular y cognitivo como en el preventivo, para evitar la acumulación de tensión y por lo tanto de dolor. Introducimos la metáfora de «relajar la vida». Planteamos la necesidad de «relajar la vida» aumentando la calidad de las relaciones personales y atendiendo a las propias necesidades emocionales.

Se realiza el primer ejercicio de relajación mediante visualización de situación agradable. Tras el ejercicio dejamos un espacio de puesta en común de las características y significado de la situación fantaseada así como de las sensaciones y dificultades en la realización del ejercicio. Sirve como ensayo de relajación y nos permite detectar las defensas y resistencias de cada paciente que redefinimos como «interferencias» normales debidas a la necesidad de encontrar el tipo de relajación adecuado a cada caso (idea de la «relajación cortada a la medida»), también resulta útil para detectar las necesidades afectivas no atendidas de cada paciente.

Al final de cada sesión damos una tarea para casa: registro de situaciones estresantes de la vida diaria auto-observando qué partes del cuerpo tienden a tensar más en estas situaciones; se prescribe la práctica de algunas técnicas que pueden ayudar a descargar tensión, como la realización de un «suspiro» o «apretar el puño», y que pueden utilizar en las situaciones ansiógenas.

- Segunda y tercera sesión: recogida y puesta en común de la tarea para casa. Cada componente del grupo explica su experiencia y dificultades fomentando la interacción grupal. Frecuentemente en este módulo ya aparece la sobreinvolucración en las responsabilidades familiares tanto de la familia nuclear como extensa y, generalmente, las diferentes experiencias comentadas nos llevan a señalar y enfatizar esta tendencia así como a analizar las repercusiones que tiene en los planos emocional y corporal.

Se realiza una revisión grupal de acontecimientos vitales estresantes fomentando el apoyo y contención emocional del grupo. Trabajamos la idea de rol femenino en la sociedad. Señalamos la tendencia a tomar una posición victimista (baja asertividad) con el consiguiente funcionamiento desadaptativo que conlleva. Se realizan ejercicios de habilidades asertivas a través de rol-playing dando tareas para casa según el caso.

Trabajamos habilidades de expresión emocional a través de la técnica del emociograma: descripción de cinco emociones básicas (tristeza, ira-enfado, miedo-ansiedad, alegría, afecto) con medición subjetiva en escala analógica, cada individuo puntúa cada emoción del 1-10 basándose en la intensidad con que la ha sentido durante las últimas semanas, se realiza puesta en común del ejercicio.

Trabajamos la insatisfacción y frustración, que muchas de ellas comparten, al analizar cómo han manejado las situaciones difíciles a lo largo de los años ofreciendo grupalmente alternativas conductuales más adaptadas. 
Prescribimos como tarea para casa hacer una lista de aquellas tareas que podrían suprimir o delegar en otros miembros de la familia.

Entregamos cinta de relajación que contiene los ejercicios de la relajación muscular progresiva sugiriendo la práctica diaria.

- Cuarta y quinta sesión: Puesta en común del listado de tareas para suprimir o delegar en los demás. Planteamiento de las dificultades para pedir ayuda o delegar tareas por los sentimientos de culpa que ello les genera. A través de la discusión y de contrastar las diferentes experiencias se va desarrollando una mayor cohesión grupal que facilita el compromiso de cambio de las componentes del grupo. Se incide en los pensamientos, sentimientos y relaciones tensionantes. Frecuentemente en estas sesiones se discute sobre los roles sociales que realizan hombres y mujeres y cómo éstos condicionan nuestras relaciones y cogniciones. Analizamos cómo estos papeles autoimpuestos inconscientemente condicionan nuestros sentimientos y pensamientos afectando al bienestar físico.

Seguimos trabajando el entrenamiento en asertividad con realización de nuevos rol-playing o esculturas familiares con psicodrama de situaciones reales planteadas por las pacientes. Recogemos la experiencia de la práctica de la relajación en casa e insistimos en la importancia de reservarse un tiempo para la práctica diaria. Prescribimos como tarea para casa realizar un escrito en el que quede reflejado cómo creen que ven la enfermedad las personas que conviven con ellas y qué creen que piensan de ella.

- Sexta y séptima: puesta en común de la tarea para casa. Recogemos diferentes ideas que aparecen recurrentemente en el discurso de las componentes del grupo acerca de lo que piensan sus familiares de la FM y que nos parecen interesantes para explicar los pensamientos y emociones que ésta genera en ellas. Por ejemplo: «No lo entienden», «Piensan que son tonterías», «Todo es de coco», «Manías», «No entienden la depresión», «Que he cambiado», «Que me alejo de mi marido», «Que paso de las cosas», «Que estoy acabando con su paciencia». Pactamos que a la próxima sesión acudirán con el familiar que convive con ellas.

La sesión con familiares involucrados se inicia con un resumen psicoeducativo sobre la fibromialgia sobre la base del mapa bio-psico-social comentado. Facilitamos el intercambio de impresiones, pensamientos y emociones que las parejas tienen acerca de la enfermedad. Se abordan distintos temas sobre la relación de pareja enmarcándolo siempre como un recurso para luchar contra la enfermedad: tiempo que dedican a la relación, dificultades con la organización familiar, mejora de actividades lúdicas en común, comunicación, relaciones afectivas y sexuales, etc. También se prescriben tareas y compromisos de cambio.

- Octava y novena sesión: A partir de dificultades que presentan en relación con los cambios planteados, se realiza abordaje a través de resolución de conflic- 
DEBATES E INFORMES

tos fomentando la participación de todos los miembros del grupo. Se señalan los rasgos perfeccionistas y autoexigentes que interfieren en el cambio de estilo de vida.

Ejercicio de relajación con sugestiones positivas sobre atender las necesidades internas y connotaciones positivas sobre los acontecimientos vitales de la vida y sacrificios realizados. Prescripción de tareas para dedicar más tiempo y esfuerzo para cuidarse a uno mismo y comprobar cómo esto mejora la autoestima y repercute positivamente en las relaciones interpersonales y bienestar físico.

- Última sesión: Puesta en común de la práctica de las pautas de la sesión anterior. Dedicamos la última sesión a que cada una de las componentes del grupo hable de cómo ha sido su experiencia en el grupo y se refuerzan los cambios realizados y la aceptación de los cambios no alcanzados redefiniéndolos positivamente. Se realiza intervención de interacción grupal resaltando los puntos fuertes de cada miembro. Se enfatizan los cambios de visión y comprensión de la fibromialgia. Comentarios que surgen reiteradamente en todos los grupos realizados y que sirven de cierre de la última sesión podemos destacar por ejemplo: «La fibromialgia no solamente es una patología de tipo muscular sino que está muy influenciada por el carácter, la manera de vivir y los problemas e insatisfacciones personales»; «la fibromialgia no se cura pero se alivia cambiando hábitos, manera de vivir y adaptándose a ella»; «lo que nos duele es la vida».

\section{Valoración y resultados}

Los resultados obtenidos, a lo largo de estos cinco años, nos llevan a valorar positivamente este tipo de intervención grupal con pacientes diagnosticadas de fibromialgia. Hemos considerado variables subjetivas como es el discurso de las propias componentes del grupo que refleja una mejor adaptación a la enfermedad, adoptando una posición más activa (por tanto, menos victimista y demandante) focalizando su atención no tanto hacia el síntoma que padecen, el dolor, sino hacia la modificación de un estilo de vida autoexigente, perfeccionista y pasivo-agresivo, tras comprobar la repercusión que esto tiene en los planos emocional y relacional e indirectamente el efecto positivo hacia el síntoma físico. Creemos también que el sentimiento de universalidad y de desingularización generado por el grupo que permite el desarrollo de la cohesión grupal facilita experiencias de aprendizaje interpersonal y de altruismo entre las componentes, que se transfieren más allá del ámbito formal del grupo y que supone para muchas de las pacientes una ampliación de la red social extra-familiar, que conlleva un importante aumento de autoestima. 
Como resultados objetivos nos basamos en las puntuaciones obtenidas en el Cuestionario de Ansiedad Estado-Rasgo (STAI) y el Inventario de Expresión de Ira Estado-Rasgo (STAXI-2). Aunque, por no ser el objetivo principal de nuestro trabajo, no nos hemos centrado en el análisis de resultados obtenidos, sí nos parece interesante comentarlos brevemente ya que nos sirven para sostener nuestra impresión subjetiva de la eficacia terapéutica del tratamiento desarrollado y aportan nueva información relevante sobre la implicación de las emociones en este trastorno.

Los resultados son los obtenidos en una muestra de dos grupos que hacen un total de veinte pacientes, con edades comprendidas entre 30 y 55 años.

El Inventario de Expresión de Ira Estado-Rasgo (STAXI-2) es un instrumento que ha probado su utilidad en distintos ámbitos de la psicología de la salud, muy especialmente en aquellos en que las emociones desempeñan un papel destacado, como son, la hipertensión, el infarto agudo de miocardio, las úlceras gástricas, el asma bronquial y el dolor (11). Asimismo en la psicología clínica es de gran interés ya que permite evaluar una variable emocional escasamente explorada, que sin embargo puede ser clave en la mayor parte de los casos que se atienden en la práctica profesional, como son, los trastornos de ansiedad, trastornos del estado de ánimo y las reacciones al estrés. El STAXI-2 mide la experiencia, expresión, control de la ira y permite identificar personas con problemas relacionados con la ira. Además permite medir el estado y rasgo de ira, y las distintas facetas de la expresión de la ira.

La ira en cuanto estado emocional psicobiológico, va unida generalmente a tensión muscular y a excitación de los sistemas neuroendocrino y nervioso autónomo, y, por tanto, este test se muestra idóneo para explorar la modulación de la ira en las fibromiálgicas y la repercusión de la ira en su salud.

El perfil dado por las puntuaciones significativas obtenidas en el pre-test (al iniciarse el grupo) nos sorprendió por la alta capacidad que tiene el test de medir y demostrar la importancia de la ira en los procesos somatizadores que se dan en el trastorno, siendo los resultados más relevantes los siguientes:

- El 78\% de las pacientes obtiene una puntuación elevada (por encima del centil 75) en la escala Rasgo de ira, que indicaría la experimentación frecuente de sentimientos de ira y frustración, indicando que la experiencia de ira se da a lo largo del tiempo y está relacionado con rasgos de personalidad.

- El 62\% obtiene una puntuación significativamente baja (por debajo del centil 25) en la escala Expresión externa de ira. Por tanto la mayoría de las pacientes a pesar de sentir altos grados de ira no la trasladan a la expresión verbal o física, no la externalizan. 
DEBATES E INFORMES

- El 70\% obtiene una puntuación significativamente alta en Expresión interna de la ira que indicaría la tendencia a suprimir los sentimientos de ira. Aquí también se muestra que a pesar de sentir intensa ira la paciente no sólo la reprime sino que la dirige hacia ella misma, favoreciendo claramente la tensión física y consecuentemente la somatización de la ira.

- El 78\% obtiene puntuación alta en la escala Control externo de la ira que indicaría la tendencia a prever y prevenir la expresión externa de la ira evitando su manifestación hacia personas u objetos del entorno.

- El 66\% obtiene una puntuación significativamente alta en la escala Control interno de la ira que mide la frecuencia con que la persona intenta controlar sus sentimientos de ira mediante la moderación y el sosiego.

- El 100\% puntúa bajo en la escala Estado de ira que mide la intensidad de los sentimientos de ira y el grado en el que el sujeto siente que está expresando su ira en un momento determinado.

Estos primeros resultados nos sorprendieron por la claridad con que describen la tendencia a reprimir la ira y el alto grado de ira que sienten de forma prolongada en el tiempo estas pacientes favoreciendo así las somatizaciones y confirmando la hipótesis de una pobre modulación de las emociones.

En las mediciones de la ira post-tratamiento se ha encontrado una leve disminución de las escalas que no llega a ser suficientemente significativa lo que apuntaría a la necesidad de incrementar el trabajo sobre la regulación de las emociones incorporando a la terapia de grupo nuevas técnicas que permitan una mejor modulación de la Ira. También realizamos medición de la ansiedad estado y rasgo antes y después del tratamiento con el conocido Cuestionario de Ansiedad EstadoRasgo (STAI). No nos detendremos a realizar un análisis tan exhaustivo con este instrumento ya que es muy utilizado en muchos estudios y no aporta información tan novedosa como el STAXI-2.

En nuestro grupo en cuanto a la relación ansiedad y fibromialgia se corrobora lo encontrado en otros estudios: una alta prevalencia tanto de la ansiedad estado como de la ansiedad rasgo en estas pacientes, siendo superior la ansiedad rasgo. Aquí el 95\% de las pacientes obtiene una puntuación muy elevada (por encima del centil 85) en la escala de ansiedad rasgo y de estado, que indicaría la experimentación frecuente de sensaciones de ansiedad.

En las medidas postratamiento sí se percibe una significativa reducción de la ansiedad estado (el 70\% pasan del centil 85 al centil 50 en cuanto a ansiedad esta$d o$ ), y una disminución ligera (no significativa) en la ansiedad rasgo, por lo que como en múltiples estudios realizados se demuestra que tanto los ejercicios de relajación y la terapia grupal favorecen la disminución de los estados de ansiedad. La baja incidencia en la ansiedad rasgo puede ser interpretada como que la ansie- 
dad rasgo es generada por aspectos estructurales de personalidad y contexto sociofamiliar que requerirían de una intervención familiar y de un periodo de tratamiento más prolongado.

\section{Conclusiones}

«Soy todo enteramente cuerpo y ninguna otra cosa. El cuerpo es una gran razón, una multitud unánime, Un estado de paz y de guerra, un rebaño y su pastor»

F. NIETZSCHE (12)

En la intervención terapéutica que hemos presentado sobre la fibromialgia no es tan importante o decisivo las técnicas específicas que se utilizan, somos plenamente concientes que otra terapia de grupo que utilice otras técnicas sería igualmente beneficiosa, para nosotros, lo decisivo es construir un marco de intervención que permita incidir en los siguientes aspectos:

A) Ofrecer una atención estructurada a la «necesidad de atención» de estas pacientes no sólo por sus características de personalidad y sociofamiliares sino por el verdadero sufrimiento que ellas padecen.

B) Partir de un enfoque psicoeducativo no dirigido tanto a aumentar la conciencia de enfermedad como se hace con los trastornos mentales severos sino más bien a lo contrario, es decir, a ayudar, respetuosamente, a estas pacientes a comprender y reconocer mejor los mecanismos psicológicos, relacionales y sociológicos a través de los cuales ésta funciona; por ejemplo, incidir en la concienciación de cómo el malestar psico-relacional encuentra vías somáticas de expresión y ofrecer mecanismos para poder expresar y «mentalizar» lo que se expresa a través de lo somático.

C) Realizar entrenamiento en habilidades de resolución de conflictos, estrategias de afrontamiento y aumento de la calidad de vida en general.

Queremos resaltar cómo en el presente estudio a través de la utilización del STAXI-2 hemos podido mostrar más «empíricamente» la importancia de la inadecuada regulación de la ira en este trastorno. Concretamente destacar que el perfil general encontrado de Expresión de la Ira de estas pacientes muestra una puntuación elevada en Rasgo de Ira y un bajo Índice de Expresión de dicha Ira, destacando un altísimo Índice de Expresión Interna de la Ira. Por tanto, estos resultados abren nuevas posibilidades de intervención psicológica y también de investigación sobre la relación entre la regulación de las emociones y la fibromialgia. 
DEBATES E INFORMES

\section{BIBLIOGRAFÍA}

(1) Freud, S., «Inhibición, síntoma y ansiedad», en Obras Completas, Madrid, Alianza.

(2) FrankL, V., Ante el vacío existencial, Barcelona, Herder, 1984.

(3) Camps, L., SÉller, D.; De Gracia, M.; ReIXACH, R., Fibromialgia y Calidad de Vida. Presentación escrita Congreso de la AENa, Girona.

(4) Generalitat de Catalunya. Departament de Salut, La fibromialgia i el síndrome de fatiga crónica, Barcelona, 2006.

(5) RAmos García, J., «Fibromialgia: ¿La histeria en el capitalismo de ficción?», Revista de la AEN, 2004, 24, 89, pp. 115-128.

(6) RENDUELES, G., «Ventajistas: de la fibromialgia a la histeria pasando por la simulación», Revista de la AEN, 2004, 24, 90, pp. 75-81.

(7) Martin, J., y otros, «Aspectos psicológicos de la fibromialgia», Revista Psiquiatría Fac. Med. Barna, 2000, 27 (1), pp. 12-18.

(8) GIMÉNEZ RiBOTA, O., y otros, «Síndrome de fibromialgia: revisión y actualización», Revista APySAM Atención Primaria y Salud Mental, 2003, 6, 4.

(9) HERnÁNDEZ HERRERA, M., «Estudio de la personalidad de los pacientes con fibromialgia», Rheuma, 1998, 3. 1994.

(10) Vinogradov, S.; Yalom, I., Guía breve de psicoterapia de grupo, Barcelona, Paidós,

(11) CASAdo, M. I., y otros, «El estudio de la ira en los trastornos cardiovasculares mediante el empleo del inventario de expresión de ira estado-rasgo», Revista de la AEN, 1997, 3, 1, pp. 5-20.

(12) Nietzsche, F., en Obras Completas, Madrid, Alianza.

* Agustín Camino Vallhonrat, psicólogo clínico. Centro de Salud Mental del Baix Empordà. Institut d'Assistència Sanitària de Girona; Beatriz Jiménez Rico, psicóloga clínica. Hospital de Día. Institut d'Assistència Sanitària de Girona; Margarita de Castro-Palomino i Serra, enfermera. Directora Adjunta de la red de salud mental del Institut d'Assistència Sanitària de Girona; Montse Fábregas, enfermera. Centro de Salud Mental del Baix Empordà. Institut d'Assistència Sanitària de Girona.

Correspondencia: Agustín Camino Vallhonrat, CSM Baix Empordà (IAS), Galeries Eurocenter, c/ Illa, 4, n. ${ }^{\circ}$ 55-61, Platja d'Aro, 17250, Girona.

** Fecha de recepción: 9-IV-2008 (aceptado el 14-VI-2008). 